\title{
Statistical Modeling of Co-Channel Interference
}

\author{
Kapil Gulati*, Aditya Chopra*, Brian L. Evans* and Keith R. Tinsley ${ }^{\dagger}$ \\ * The University of Texas at Austin, Austin, Texas 78712 \\ Email: \{gulati,chopra,bevans\} @ece.utexas.edu \\ ${ }^{\dagger}$ Intel Corporation, Santa Clara, CA 95054 \\ Email: keith.r.tinsley@intel.com
}

\begin{abstract}
With increasing spatial reuse of the radio spectrum, co-channel interference is becoming the dominant noise source and may severely degrade the communication performance of wireless transceivers. In this paper, we consider the problem of statistical-physical modeling of co-channel interference. Statistical modeling of interference is a useful tool to analyze the outage probabilities in wireless networks and to design interference-aware transceivers. Our contributions include (1) developing a unified framework to derive interference models for various wireless network environments, (2) demonstrating the applicability of the symmetric alpha stable and Middleton Class A distributions in modeling co-channel interference in ad-hoc and cellular network environments, and (3) deriving analytical conditions on the system model parameters for which these distributions accurately model the statistical properties of the interference. Simulation results allow us to compare the key properties of empirical co-channel interference and their statistical models under different wireless network environments.
\end{abstract}

\section{INTRODUCTION}

Current and future wireless communication systems require higher spectral usage due to increasing demand in user data rates. One of the principal techniques for efficient spectral usage is to implement a dense spatial reuse of the available radio spectrum. This causes severe co-channel interference, which limits the system performance. Knowledge of the interference statistics is integral to analyzing performance of wireless networks, including outage probability and throughput, and can also be used to design transceivers with improved communication performance [1], [2].

Statistical modeling of co-channel interference has been extensively studied in literature, for both fixed topology wireless networks [3] and random Poisson interference fields [4], [5]. The statistical techniques used in modeling interference include empirical methods and statistical-physical methods. Empirical approaches, such as using the Gaussian mixture distribution [6], fit a mathematical model to measured received signals, without regard to the physical generation mechanisms behind the interference. Statistical-physical models, on the other hand, model interference based on the physical principles that govern generation and propagation of the interferencecausing emissions. Statistical-physical models thus tend to be more accurate than empirical models.

Key statistical-physical models include the symmetric alpha stable model [7] and the Middleton Class A, B and C models

This research was supported by Intel Corporation.
[8]. The symmetric alpha stable model has been widely used to model co-channel interference in a Poisson field of narrowband interferers in ad-hoc network environments [4], [5], [9]. Middleton models have been derived under a wider range of physical conditions that include narrowband and broadband interference emissions and transients at the receiver. They also model the background thermal noise present at the receivers [8]. Middleton models, however, have not been widely used to characterize the co-channel interference in wireless network environments.

One of the key contributions of this paper is to develop a unified framework to derive the co-channel interference statistics in different wireless network environments and establish the applicability of the symmetric alpha stable and Middleton Class A model in ad-hoc and cellular network environments. Analytical constraints on the system model parameters for which these distributions accurately model the statistical properties of the interference are also derived. When exact statistics cannot be derived in closed-form, the paper focuses on accurately modeling the tail probability of the interference distribution.

We will use the following notation throughout this paper. Random variables are represented using boldface notation, deterministic parameters are represented using non-boldface type, $\mathbb{E}_{\mathbf{X}}\{f(\mathbf{X})\}$ denotes the expectation of the function $f(\mathbf{X})$ with respect to the random variable $\mathbf{X}$, and $\mathbb{P}(\cdot)$ denotes the probability of a random event.

\section{System MODEL}

Consider a wireless communication system in which the receiver receives a signal of interest in the presence of interfering signals. The interfering users are assumed to be on the same plane as the receiver, concentrated on a spatial interference space $\Gamma\left(r_{l}, r_{h}\right)$, and be potentially infinite in number. The receiver is assumed to employ a single omnidirectional antenna and is located at a distance $r_{m}$ from the origin of the two-dimensional coordinate system. The parametric interference space $\Gamma\left(r_{l}, r_{h}\right)$ is defined as

$$
\Gamma\left(r_{l}, r_{h}\right)=\left\{x \in \mathbb{R}^{2}: r_{l} \leq\|x\| \leq r_{h}\right\}
$$

where $\|\cdot\|$ denotes the Euclidean norm. The three-dimensional scenario, in which the interferers are located in a volume space around the receiver, is a straightforward extension to the twodimensional scenario considered in this paper. 


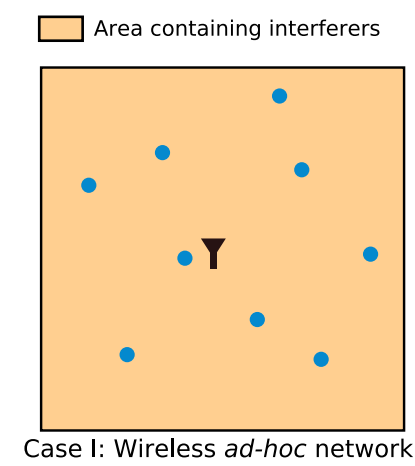

Case I: Wireless ad-hoc network

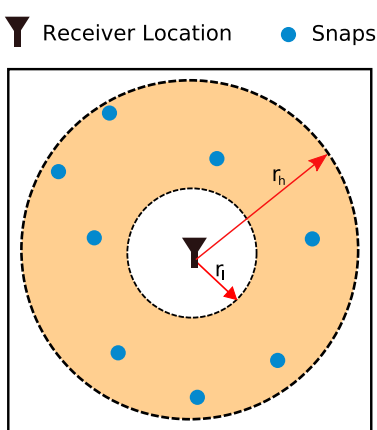

Case II: Cellular Network (Base Station)

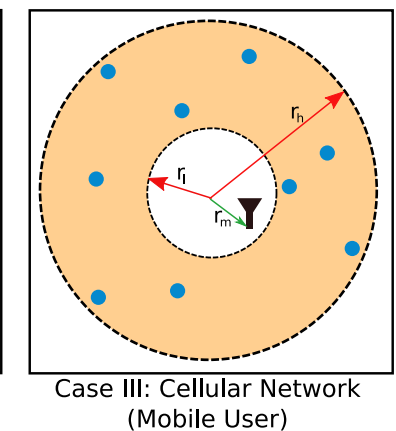

(Mobile User)

Fig. 1: Interference space and receiver location for different wireless network environments.

At each sampling time instant $n$, the number of active interferers are assumed to be distributed according to a spatial Poisson process on the space $\Gamma$ with density $\lambda$. As shown in [4], this model is sufficient to capture both the emerging interferers, whose contributions arrive at the receiver for the first time at the time instant $n$, and interferers that first emerged at some prior sampling time instant $m<n$ but are still active till the sample time $n$. Further, we assume that the interferers transmit in an uncoordinated manner, which is required for the Poisson assumption to be valid.

The baseband model for the sum interference $\mathbf{Y}$ at the receiver at any time instant can then be represented as

$$
\mathbf{Y}=\sum_{i=1}^{\mathbf{K}} \mathbf{r}_{i}^{-\frac{\gamma}{2}} \mathbf{h}_{i} \mathbf{X}_{i}
$$

where $\mathbf{K}$ is the random number of active interferers at that time instant, $i$ is the interferer index, $\mathbf{r}_{i}$ is the random distance of active interferers from the receiver, $\gamma$ is the power pathloss exponent, $\mathbf{h}_{i}$ is the independent and identically distributed (i.i.d.) random fast fading experienced by each interferer emission, and $\mathbf{X}_{i}$ are the random baseband emissions from the active interferers.

We assume that all potential interferers have i.i.d. symmetric narrowband emissions of the form [10]

$$
\mathbf{X}_{i}=\mathbf{B}_{i} \cos \left(\phi_{i}\right)
$$

where $\mathbf{B}_{i}$ is the i.i.d. envelope, and $\phi_{i}$ is the i.i.d. random phase of the emissions. Further, we assume that the emerging times of the interferers are uniformly distributed between the sampling times at the receiver. Thus the phase $\phi_{i}$ of the emissions at the sampling instants can be assumed to uniformly distributed between $[0,2 \pi]$. The assumption of i.i.d. emissions is valid for wireless communication networks without power control and may not be true for modeling interference from diverse types of interferers with unequal transmit power (e.g. base stations and mobile users).

\section{Statistical Modeling}

In this section, we derive the characteristic function of the co-channel interference. We establish a unified framework and derive the symmetric alpha stable model and the
Middleton Class A model (without the Gaussian component) for interference in ad-hoc and cellular network environments. The characteristic function of the sum interference $\mathbf{Y}$ can be expressed as

$$
\begin{aligned}
& \Phi_{\mathbf{Y}}(\omega)=\mathbb{E}_{\mathbf{r}_{i}, \mathbf{h}_{i}, \mathbf{X}_{i}, \mathbf{K}}\left\{e^{\left.j \omega \sum_{i=1}^{\mathbf{K}} \mathbf{r}_{i}^{-\frac{\gamma}{2}} \mathbf{h}_{i} \mathbf{X}_{i}\right\}}\right. \\
&=\sum_{k=0}^{\infty} \mathbb{E}\left\{e^{j \omega \sum_{i=1}^{k} \mathbf{r}_{i}^{-\frac{\gamma}{2}} \mathbf{h}_{i} \mathbf{X}_{i}} \mid k \text { in } \Gamma\left(r_{l}, r_{h}\right)\right\} \times \\
& \mathbb{P}\left(k \text { in } \Gamma\left(r_{l}, r_{h}\right)\right) .
\end{aligned}
$$

Conditioned on the number of interferers present in the space $\Gamma\left(r_{l}, r_{h}\right)$, the interferer locations are mutually independent and uniformly distributed across this space [7]. Henceforth, we remove the conditioning on the number of interferers from the expectation by noting that the interferers are uniformly distributed in the space $\Gamma\left(r_{l}, r_{h}\right)$. Further, in the absence of power control, the emission from the interferers can be assumed to be independent and identically distributed. The characteristic function can be expressed as

$$
\begin{aligned}
& \Phi_{\mathbf{Y}}(\omega)=\sum_{k=0}^{\infty}\left[\mathbb{E}\left\{e^{j \omega \mathbf{r}^{-\frac{\gamma}{2}} \mathbf{h X}}\right\}\right]^{k} \times \\
& \frac{\left[\lambda \pi\left(r_{h}^{2}-r_{l}^{2}\right)\right]^{k} e^{-\lambda \pi\left(r_{h}^{2}-r_{l}^{2}\right)}}{k !} \\
&=e^{\lambda \pi\left(r_{h}^{2}-r_{l}^{2}\right)\left(\mathbb{E}\left\{e^{j \omega \mathbf{r}-\frac{\gamma}{2} \mathbf{h x}}\right\}-1\right)} .
\end{aligned}
$$

By taking the logarithm of $\Phi_{Y}(\omega)$, and noting that $\mathbf{X}=$ $\mathbf{B} \cos (\phi)$, the $\log$-characteristic function is

$\psi_{\mathbf{Y}}(\omega)=\lambda \pi\left(r_{h}^{2}-r_{l}^{2}\right)\left(\mathbb{E}_{\mathbf{r}, \mathbf{h}, \mathbf{B}, \phi}\left\{e^{j \omega \mathbf{r}^{-\frac{\gamma}{2}} \mathbf{h B} \cos (\phi)}\right\}-1\right)$.

By using the identity

$$
e^{j a \cos (\phi)}=\sum_{k=0}^{\infty} j^{k} \epsilon_{k} J_{k}(a) \cos (k \phi)
$$

where $\epsilon_{0}=1, \epsilon_{k}=2$ for $k \geq 1$, and $J_{k}(\cdot)$ denotes the Bessel function of order $k$, the log-characteristic function can 
be expressed as

$$
\begin{aligned}
\psi_{\mathbf{Y}}(\omega)=\lambda \pi\left(r_{h}^{2}-r_{l}^{2}\right) \times \\
\left(\mathbb{E}\left\{\sum_{k=0}^{\infty} j^{k} \epsilon_{k} J_{k}\left(\omega \mathbf{r}^{-\frac{\gamma}{2}} \mathbf{h B}\right) \cos (k \boldsymbol{\phi})\right\}-1\right) .
\end{aligned}
$$

Since $\phi$ is assumed to be uniformly distributed between 0 and $2 \pi, \mathbb{E}_{\phi}\{\cos (k \phi)\}=0$ for $k \geq 1$, and (10) reduces to

$$
\psi_{\mathbf{Y}}(\omega)=\lambda \pi\left(r_{h}^{2}-r_{l}^{2}\right)\left(\mathbb{E}_{\mathbf{r}, \mathbf{h}, \mathbf{B}}\left\{J_{0}\left(\omega \mathbf{r}^{-\frac{\gamma}{2}} \mathbf{h B}\right)\right\}-1\right) .
$$

The log-characteristic function derived in (11) holds in general for any space of narrowband interferers governed by the parameters $r_{h}$ and $r_{l}$ and the receiver location $r_{m}$. The receiver location $r_{m}$ affects the expectation in (11). We now consider the following two cases and further simplify the logcharacteristic function.

\section{A. Ad-hoc network (Case I): $r_{l}=0, r_{h} \rightarrow \infty$}

Consider an ad-hoc network, as shown in Fig. 1, where the interfering sources are distributed according to a spatial Poisson point process on the plane. Note that $r_{m}$ can be assumed to be 0 without any loss in generality of the result. We consider the interference space $\Gamma\left(0, r_{h}\right)$ and take the limit on the log-characteristic function as $r_{h} \rightarrow \infty$ [9]. Recall that the expectation in (11) is conditioned such that the interferer locations are uniformly distributed in the space $\Gamma$. The distance of the interferer from the receiver thus follows the distribution

$$
f_{\mathbf{r}}(r)= \begin{cases}\frac{2 r}{r_{h}^{2}} & \text { if } 0 \leq r \leq r_{h} \\ 0 & \text { if } r>r_{h}\end{cases}
$$

Expanding the expectation in (11), we have

$$
\begin{array}{r}
\psi_{\mathbf{Y}}(\omega)=\lim _{r_{h} \rightarrow \infty} \lambda \pi r_{h}^{2}\left(\int_{0}^{r_{h}} \mathbb{E}_{\mathbf{h}, \mathbf{B}}\left\{J_{0}\left(\omega \mathbf{r}^{-\frac{\gamma}{2}} \mathbf{h B}\right)\right\} \frac{2 r}{r_{h}^{2}} d r\right. \\
-1) .
\end{array}
$$

Integrating the above by parts, taking the limit as $r_{h} \rightarrow \infty$, and noting that $\lim _{r_{h} \rightarrow \infty} \mathbb{E}_{\mathbf{B}, \mathbf{h}}\left\{r_{h}^{2}\left(J_{0}\left(w r_{h}^{-\frac{\gamma}{2}} \mathbf{B h}\right)-1\right)\right\}=0$, we have

$$
\begin{aligned}
\psi_{\mathbf{Y}}(\omega) & =|w|^{\frac{4}{\gamma}} \lambda \pi \mathbb{E}_{\mathbf{h}, \mathbf{B}}\left\{\mathbf{h}^{\frac{4}{\gamma}} \mathbf{B}^{\frac{4}{\gamma}}\right\} \int_{0}^{\infty} \frac{J_{0}^{\prime}(x)}{x^{\frac{4}{\gamma}}} d x \\
& =-|w|^{\frac{4}{\gamma}} \lambda \pi \mathbb{E}_{\mathbf{h}, \mathbf{B}}\left\{\mathbf{h}^{\frac{4}{\gamma}} \mathbf{B}^{\frac{4}{\gamma}}\right\} \int_{0}^{\infty} \frac{J_{1}(x)}{x^{\frac{4}{\gamma}}} d x .
\end{aligned}
$$

Equation (14) is the log-characteristic function of a symmetric alpha stable distribution centered at zero such that

$$
\psi_{\mathbf{Y}}(w)=-\sigma|\omega|^{\alpha}
$$

where $\alpha=\frac{4}{\gamma}$ is the characteristic exponent $(0<\alpha \leq 2)$, and $\sigma=\lambda \pi \mathbb{E}_{\mathbf{h}, \mathbf{B}}\left\{\mathbf{h}^{\alpha} \mathbf{B}^{\alpha}\right\} \int_{0}^{\infty} \frac{J_{1}(x)}{x^{\alpha}}$ is the dispersion parameter of the symmetric alpha stable distribution [7], [9]. Hence, when the interferers are distributed according to a spatial Poisson process on the entire plane, the co-channel interference follows a symmetric alpha stable distribution.
B. Cellular network (Cases II and III): $r_{l}>0, r_{h}<\infty$, $r_{m}<r_{l}<r_{h}$

Consider a cellular network, as shown in Fig. 1, where all the interferers are outside the cell in which the desired receiver is present $\left(r_{l}>0\right)$. Further, we consider the interferers within a maximum distance beyond which the interferers do not generate significant interference $\left(r_{h}<\infty\right)$. In [10], Middleton proposed an approximation of the log-likelihood function for $\omega$ in the neighborhood of zero. From Fourier analysis, the behavior of the characteristic function in the neighborhood of zero governs the tail probabilities of the random variable. The proposed approximation is based on the following identity [10]:

$\mathbb{E}_{\mathbf{r}, \mathbf{h}, \mathbf{B}}\left\{J_{0}\left(\omega \mathbf{r}^{-\frac{\gamma}{2}} \mathbf{h B}\right)\right\}=e^{-\frac{w^{2} \mathbb{E}_{\mathbf{r}, \mathbf{h}, \mathbf{B}}\left\{\mathbf{r}^{-\gamma} \mathbf{h}^{2} \mathbf{B}^{2}\right\}}{4}}\left(1+\Theta\left(w^{4}\right)\right)$

Here, $\Theta\left(w^{4}\right)$ indicates a correction term with the lowest exponent in $\omega$ of four and is given by (19). Using this identity, the log-characteristic function in (11) can be expressed as

$$
\psi_{\mathbf{Y}}(\omega)=\lambda \pi\left(r_{h}^{2}-r_{l}^{2}\right)\left(e^{-\frac{w^{2} \mathbb{E}_{\mathbf{r}, \mathbf{h}, \mathbf{B}}\left\{\mathbf{r}^{\left.-\gamma_{\mathbf{h}} \mathbf{B}^{2}\right\}}\right.}{4}}-1\right) .
$$

Equation (17) is the log-characteristic function of a Middleton Class A distribution (without the additive Gaussian component) such that

$$
\psi_{\mathbf{Y}}(w)=A\left(e^{-\frac{w^{2} \Omega_{2 A}}{2 A}}-1\right)
$$

where $A=\lambda \pi\left(r_{h}^{2}-r_{l}^{2}\right)$ is the overlap index that indicates the amount of impulsiveness of the interference, and $\Omega_{2 A}=$ $\frac{A \times \mathbb{E}_{\mathbf{r}, \mathbf{h}, \mathbf{B}}\left\{\mathbf{r}^{-\gamma} \mathbf{h}^{2} \mathbf{B}^{2}\right\}}{2}$ is the mean intensity of the interference [8]. Hence, the co-channel interference where the interferers are distributed according to a spatial Poisson process on the space $\Gamma\left(r_{l}, r_{h}\right)$, when $0<r_{l}<r_{h}<\infty$, as observed at the base station $\left(r_{m}=0\right)$ or the mobile user $\left(0<r_{m}<r_{l}\right)$ follows the Middleton Class A distribution. It should be emphasized that the correspondence to the Middleton Class A distribution is particularly valid for modeling the tail probabilities.

The approximation in (16) and the subsequent interference model in (18) is valid for $r_{l}>0$ and $r_{m}<r_{l}$, since $\Omega_{2 A} \rightarrow \infty$ as $r_{l} \rightarrow 0$ or as $r_{m} \rightarrow r_{l}$; which is not physical since the mean intensity is always finite in this case. This is unlike Case $I$ in Section III-A where the interference was modeled for $r_{l}=0$. This is the key difference between the symmetric alpha stable and Middleton Class A models for interference.

As $r_{h} \rightarrow \infty$, the overlap index $A \rightarrow \infty$ also, while the mean intensity $\Omega_{2 A}$ of the interference is finite. From (18), it can be shown that the Middleton Class A model is equivalent to a Gaussian model as $A \rightarrow \infty$. The Gaussian model, however, may be an inaccurate model for the sum interference in this scenario. This is due to the fact that the $\Theta\left(\omega^{4}\right)$ correction term in (16) cannot be ignored as $r_{h} \rightarrow \infty$. The parameter $r_{h}$, however, can be bounded above in magnitude because the 
interference power cannot be observed if its intensity is lower than the receiver noise floor [10].

Next, we quantify the range of the system model parameters over which the Middleton Class A model provides an accurate approximation to the co-channel interference in cellular network environments. The $\Theta\left(\omega^{4}\right)$ correction term in (16) can be expressed as [10]

$$
\Theta\left(\omega^{4}\right)=\sum_{k=2}^{\infty} \frac{\left(\mathbb{E}_{\mathbf{Z}}\{\mathbf{Z}\}\right)^{k} w^{2 k}}{2^{2 k} k !} \mathbb{E}_{\mathbf{Z}}\left\{{ }_{1} F_{1}\left(-k ; 1 ; \frac{\mathbf{Z}}{\mathbb{E}_{\mathbf{Z}}\{\mathbf{Z}\}}\right)\right\}
$$

where the random variable $\mathbf{Z}=\mathbf{r}^{-\gamma} \mathbf{h}^{2} \mathbf{B}^{2}$, and ${ }_{1} F_{1}(a ; b ; x)$ is the confluent hypergeometric function of the first kind. From (16), a first-order measure of the accuracy of the approximation can be expressed by comparing the coefficient of the $\omega^{4}$ term in $e^{-\frac{w^{2} \mathbb{E}_{\mathbf{r}, \mathbf{h}, \mathbf{B}}\left\{\mathbf{r}^{-\gamma} \mathbf{h}^{2} \mathbf{B}^{2}\right\}}{4}}$ against the coefficient of the $\omega^{4}$ in the correction term $\Theta\left(\omega^{4}\right)$. Using the fact that

$$
{ }_{1} F_{1}(-2 ; 1 ; x)=\frac{1}{2}\left(x^{2}-4 x+2\right),
$$

the coefficient of $\omega^{4}$ in the correction term (i.e. $c_{4}$ ) can be expressed as

$$
c_{4}=\frac{\mathbb{E}_{\mathbf{Z}}\left\{\mathbf{Z}^{2}\right\}-2\left[\mathbb{E}_{\mathbf{Z}}\{\mathbf{Z}\}\right]^{2}}{128} .
$$

Thus, the Middleton Class A model provides a good approximation when the system model parameters, such as $r_{h}, r_{l}, r_{m}$, and $\gamma$ satisfy

$$
\Rightarrow\left|\frac{\mathbb{E}_{\mathbf{Z}}\left\{\mathbf{Z}^{2}\right\}-2\left[\mathbb{E}_{\mathbf{Z}}\{\mathbf{Z}\}\right]^{2}}{128}\right|<<\frac{\left[\mathbb{E}_{\mathbf{Z}}\{\mathbf{Z}\}\right]^{2}}{32}
$$

A desirable attribute of the Middleton Class A model is the existence of a closed-form expression for the probability density function. Using the log-characteristic function in (18), the probability density function can be expressed as

$$
f_{\mathbf{Y}}(y)=e^{-A} \delta(y)+\sum_{m=1}^{\infty} \frac{e^{-A} A^{m}}{m !} \frac{e^{-\frac{y^{2}}{2 \sigma_{m}^{2}}}}{\sqrt{2 \pi} \sigma_{m}}
$$

where $\sigma_{m}^{2}=\frac{m}{A} \Omega_{2 A}$ and $\delta(\cdot)$ denotes the Dirac delta functional.

\section{TAIL Probabilities}

In this section, we give the analytical expressions for the tail probabilities of the three interference models discussed in the paper. The tail probability of a symmetric alpha stable random variable with parameters $\alpha$ and $\sigma$ is given as [7]

$$
\mathbb{P}_{S \alpha S}(\mathbf{Y}>y)=\frac{y^{-\alpha} \sigma^{\alpha} C(\alpha)}{2}
$$

as $y \rightarrow \infty$, where

$$
C(\alpha)= \begin{cases}\frac{2}{\pi} & \text { when } \alpha=1, \\ \frac{1-\alpha}{\Gamma(2-\alpha) \cos \left(\frac{\pi \alpha}{2}\right)} & \text { otherwise. }\end{cases}
$$

Using (24), the tail probability for the Middleton Class A distribution with parameters $A$ and $\Omega_{2 A}$ can be expressed as

$$
\mathbb{P}_{M C A}(\mathbf{Y}>y)=\sum_{m=1}^{\infty} \frac{e^{-A} A^{m}}{m !} \frac{1}{2} \operatorname{erfc}\left(\frac{y}{\sqrt{\frac{2 m \Omega_{2 A}}{A}}}\right)
$$

for $y>0$. Here $\operatorname{erfc}(x)=\frac{2}{\sqrt{\pi}} \int_{x}^{\infty} e^{-t^{2}} d t$ represents the complementary error function. The tail probability for a Gaussian random variable with variance $\sigma^{2}$ can be expressed as

$$
\mathbb{P}_{G A U S S}(\mathbf{Y}>y)=\frac{1}{2} \operatorname{erfc}\left(\frac{y}{\sqrt{2 \sigma^{2}}}\right) \text {. }
$$

\section{RESUlts}

Using the physical model discussed in Section II, we apply Monte-Carlo numerical techniques to simulate the co-channel interference observed at the receiver in various wireless network environments. At each sample instant, the location of the active interferers is generated as a realization of a twodimensional spatial Poisson point process with intensity $\lambda=$ $10^{-4}$ within the region $\Gamma\left(r_{l}, r_{h}\right)$. Parameter values for $r_{l}, r_{h}$ and $r_{m}$ change according to the wireless network model under consideration. It should be noted that parameters denoting distance are are treated as dimensionless quantities as this does not influence the statistics of the resultant interference.

From (2), the interference observed at the receiver at each time instant is a sum of narrowband emissions, as given by (3), from the active interferers in $\Gamma\left(r_{l}, r_{h}\right)$ which have experienced propagation pathloss and fast-fading. In our simulations, we assume a power path-loss exponent $\gamma=4$ and Rayleigh fading (h) with unit energy. The amplitude of the interferer emissions, B, was chosen as a constant for a particular wireless environment such that the tail probability, $\mathbb{P}(\mathbf{Y}>y)$, at an interference threshold of $y=10$ is of the order of $10^{-5}$. The probability distribution of co-channel interference is empirically estimated from 500000 time samples of the received interference using kernel smoothed density estimators.

Accuracy of the statistical-physical models for co-channel interference is established by comparing the empirical and interference model tail probabilities. We compare the asymptotic decay rates of the tail probabilities given by

$$
\rho(y)=-\frac{\log (\mathbb{P}(\mathbf{Y}>y))}{y}
$$

where $\rho(y)$ is the asymptotic decay rate at interference amplitude $y$. The decay rate is the rate at which the tail probability asymptotically approaches zero. Impulsive distributions have smaller decay rates as compared to non-impulsive distributions and are hence referred to as heavy-tailed distributions. The decay rates are a useful metric to compare the extreme value statistics of different statistical models with respect to the empirically estimated distribution.

Following are the results comparing symmetric alpha stable and Middleton Class A distributions against the empirical distribution of co-channel interference for each of the wireless network environment described in Section III. Further, we 


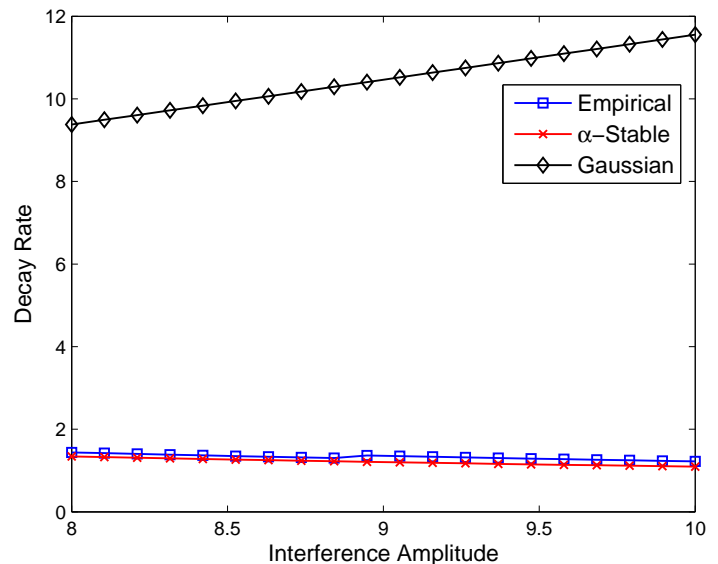

Fig. 2: Decay rates for simulated and interference model distributions in Case I $\left(r_{h}=\infty, r_{l}=0, \mathbf{B}=2\right)$.

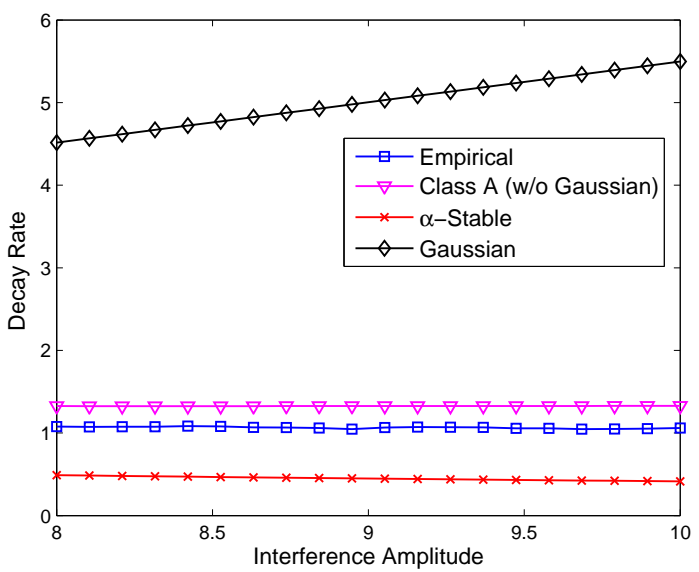

Fig. 3: Decay rates for simulated and interference model distributions in Case II-a $\left(r_{h}=40, r_{l}=20, \mathbf{B}=1800\right)$.

compare the empirical distribution of co-channel interference to a Gaussian distribution with equal variance for all scenarios.

\section{A. Ad-hoc network (Case I): $r_{l}=0, r_{h} \rightarrow \infty$}

Case I describes the wireless ad-hoc network model, where the interferer are located in the region $\Gamma\left(0, r_{h}\right)$ and distributed as a spatial Poisson process with intensity $\lambda$. In Section III, we have shown that the resulting interference would have a symmetric alpha stable distribution. Fig. 2 shows the decay rates of the empirical distribution compared with the statistical models. The empirical distribution indeed matches closely to an alpha stable distribution in terms of decay rates. Note that the Middleton Class A model is not suitable in this case as $\Omega_{2 A} \rightarrow \infty$ (as discussed in Section III).

\section{B. Cellular network (Case II): $r_{l}>0, r_{h}<\infty, r_{m}=0$}

Case II describes the cellular network model where the receiver is located at the center of the cell. We assume that the base station employs time or frequency allocation amongst the users and hence no interferers are present within

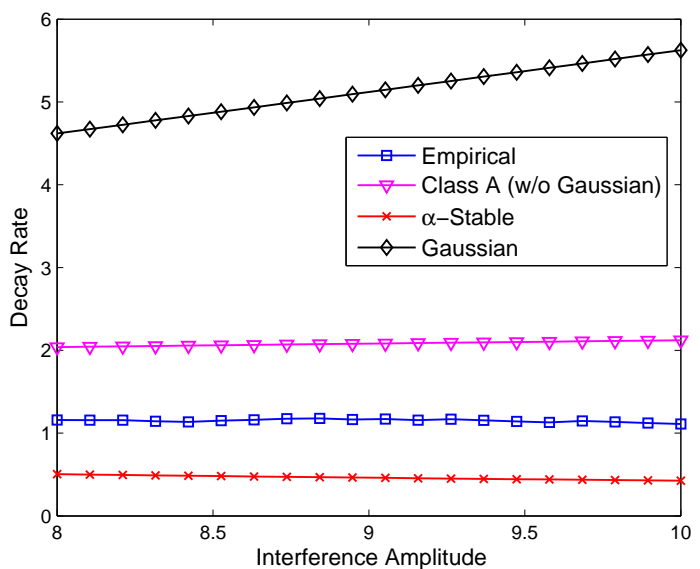

Fig. 4: Decay rates for simulated and interference model distributions in Case II-b $\left(r_{h}=80, r_{l}=20, \mathbf{B}=1600\right)$.

TABLE I: Kulback-Liebler divergence between empirical and model distribution in different wireless network scenarios.

\begin{tabular}{|c|c|c|c|}
\hline & \multicolumn{3}{|c|}{ Model Distribution } \\
\hline $\begin{array}{c}\text { Wireless network } \\
\text { scenario }\end{array}$ & $\alpha$-stable & $\begin{array}{c}\text { Class A } \\
\text { (w/o Gaussian term) }\end{array}$ & Gaussian \\
\hline Case I & 0.0032 & - & 4.1727 \\
\hline Case II-a & 0.1993 & 0.0069 & 0.5628 \\
\hline Case II-b & 0.0548 & 0.0914 & 0.2068 \\
\hline Case III-a & 0.1804 & 0.0089 & 0.5733 \\
\hline Case III-b & 0.0992 & 0.1369 & 0.3376 \\
\hline
\end{tabular}

the cell. We place the receiver at the origin of our twodimensional space and the interferers are located within the region $\Gamma\left(r_{l}, r_{h}\right)$ such that $r_{l}>0, r_{h}<\infty$. In Section III, we show that the probability density function at the extreme values of the resultant interference in this environment is modeled well using the Middleton Class A distribution. The model is accurate only for certain ranges of the system parameters $r_{l}$ and $r_{h}$ as given by (23). Case II is sub-divided into two scenarios: Case II-a and Case II-b. In Case II-a, the simulation parameters are within range of values given by (23) with $r_{l}$ and $r_{h}$ as 20 and 40, respectively. Fig. 3 shows the decay rates of co-channel interference generated in this wireless network are approximated well by the Middleton Class A. For these values of parameters $r_{l}$ and $r_{h}$, the higher-order terms in the characteristic function from (18) are negligible. In Case II$\mathrm{b}$, the effect of the higher-order terms in the characteristic function is illustrated by choosing parameter values $r_{l}$ and $r_{h}$ as 20 and 80 , respectively. Fig. 4 shows that the Middleton model from (24) is no longer a good approximation to the density function of received interference.

\section{Cellular network (Case III): $r_{l}>0, r_{h}<\infty, r_{m}<r_{l}$}

Case III also describes a cellular network, but unlike Case II the receiver is located away from the center of the cell. The distance of the receiver from the cell center (origin) is given by $r_{m}$. We simulate co-channel interference for two scenarios: in case III-a the receiver is located close to the cell center $\left(r_{m}<<r_{l}\right)$, and in case III-b the receiver is 
TABLE II: Statistical-physical modeling of co-channel interference in different wireless network environments.

\begin{tabular}{|c|c|c|}
\hline & Symmetric Alpha Stable & Middleton Class A (w/o Gaussian component) \\
\hline $\begin{array}{l}\text { 1. Statistical-physical model for } \\
\text { interference in }\end{array}$ & $\begin{array}{cl}\text { a. } & \text { wireless ad-hoc network environment }\left(r_{l}=0 \text {, }\right. \\
& \left.r_{h} \rightarrow \infty\right) \\
\text { b. } & \text { wireless cell-edge users in cellular network } \\
& \text { environment }\left(r_{m} \rightarrow r_{l}, r_{h} \rightarrow \infty\right)\end{array}$ & $\begin{array}{ll}\text { a. } & \text { wireless cellular network environment }\left(r_{m}<\right. \\
& \left.r_{l}<r_{h}, r_{l}>0, r_{h}>>r_{l}\right)\end{array}$ \\
\hline 2. Statistical property modeled & exact statistics & tail probability \\
\hline 3. Accurate model for & $r_{l}=0$ or $r_{m}=r_{l}$, and $r_{h}>>r_{l}$ & when conditions in (23) are met \\
\hline 4. Additive thermal noise & Middleton Class B (narrowband) model [8] & Middleton Class A model \\
\hline
\end{tabular}

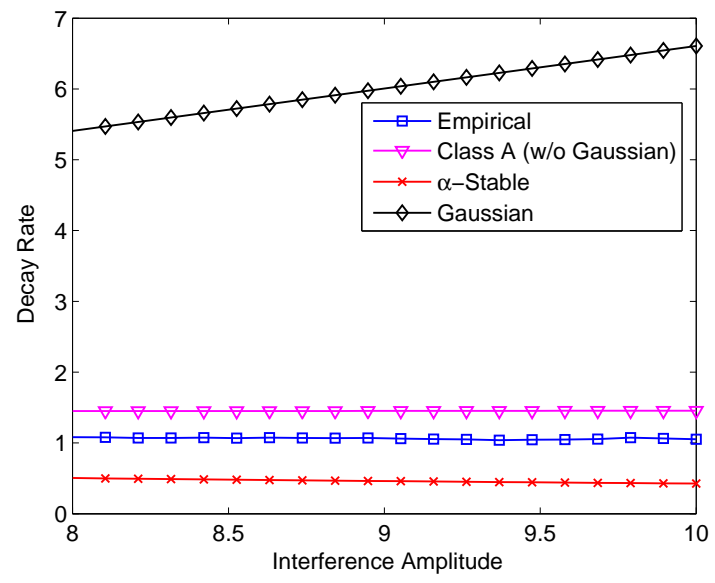

Fig. 5: Decay rates for simulated and interference model distributions in Case III-a $\left(r_{h}=40, r_{l}=20, r_{m}=3, \mathbf{B}=1600\right)$.

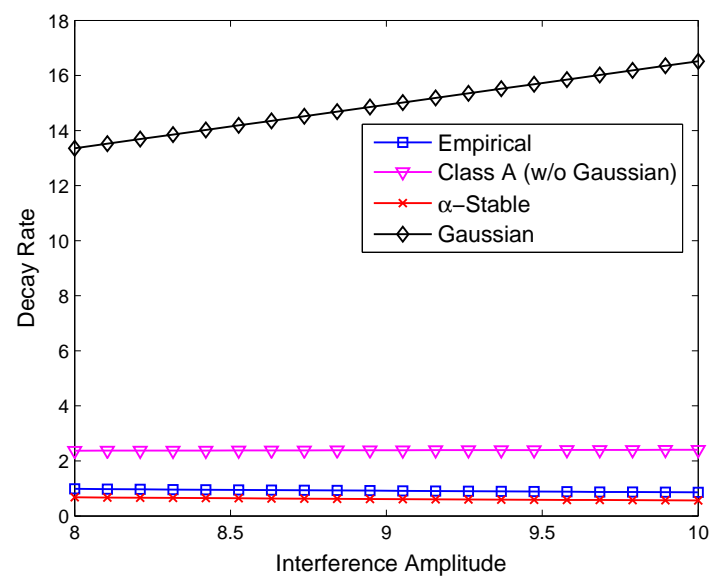

Fig. 6: Decay rates for simulated and interference model distributions in Case III-b $\left(r_{h}=40, r_{l}=20, r_{m}=15, \mathbf{B}=400\right)$.

located close to the edge of the cell $\left(r_{m} \rightarrow r_{l}\right)$. Figs. 5 and 6 show the corresponding decay rates for cases III-a and III$\mathrm{b}$, respectively. The parameter $r_{m}$ has the value 3 and 15 for scenarios III-a and III-b, respectively. Fig. 5 shows that when the receiver is closer to the cell center, the resulting interference distribution has decay rates similar to those of the Middleton Class A distribution. Fig. 6 shows that when the receiver is closer to the cell edge, the decay rates of the empirical interference distribution resemble those of an alpha stable distribution.
Another method to quantify the accuracy of fit of a statistical model is to use the Kulback-Liebler (KL) divergence [11], where a KL-divergence of zero indicates an exact match of the densities. Table I lists the KL-divergence values between empirical and model distributions for different network environments. Correspondence in tail probabilities does not imply a lower KL-divergence, since the KL-divergence finds the relative error between two distribution functions over their entire support. Thus, while the alpha stable model has a relatively low KL-divergence with respect to the other statistical models, it may still be an inaccurate model for modeling the extreme statistics (as in Case II and Case III-a).

In all of the network models discussed above, the statistics of co-channel interference are not modeled well by the Gaussian distribution, particularly at high interference amplitudes. The Gaussian distribution decays far too quickly to model the impulsive nature of co-channel interference accurately.

Key insights from this paper are summarized in Table II.

\section{REFERENCES}

[1] S. Weber, J. G. Andrews, and N. Jindal, "The effect of fading, channel inversion, and threshold scheduling on ad hoc networks," IEEE Transactions on Information Theory, vol. 53, no. 11, pp. 4127-4149, 2007.

[2] K. Gulati, A. Chopra, R. W. Heath, B. L. Evans, K. R. Tinsley, and $X$. E. Lin, "MIMO receiver design in the presence of radio frequency interference," in Proc. IEEE Global Communications Conference, Nov. 30-Dec. 42008.

[3] R. Prasad and A. Kegel, "Improved assessment of interference limits in cellular radio performance," IEEE Transactions on Vehicular Technology, vol. 40, pp. 412-419, May 1991.

[4] X. Yang and A. Petropulu, "Co-channel interference modeling and analysis in a Poisson field of interferers in wireless communications," IEEE Transactions on Signal Processing, vol. 51, no. 1, pp. 64-76, 2003.

[5] J. Ilow and D. Hatzinakos, "Analytic alpha-stable noise modeling in a Poisson field of interferers or scatterers," IEEE Transactions on Signal Processing, vol. 46, no. 6, pp. 1601-1611, June 1998.

[6] R. S. Blum, R. J. Kozick, and B. Sadler, "An adaptive spatial diversity receiver for non-Gaussian interference and noise," IEEE Transactions on Signal Processing, vol. 47, no. 8, pp. 2100-2111, Aug. 1999.

[7] G. Samorodnitsky and M. S. Taqqu, Stable Non-Gaussian Random Processes: Stochastic Models with Infinite Variance. Chapman and Hall, New York, 1994.

[8] D. Middleton, "Non-Gaussian noise models in signal processing for telecommunications: New methods and results for class A and class B noise models," IEEE Transactions on Information Theory, vol. 45, no. 4, pp. 1129-1149, May 1999.

[9] E. S. Sousa, "Performance of a spread spectrum packet radio network link in a Poisson field of interferers," IEEE Transactions on Information Theory, vol. 38, no. 6, pp. 1743-1754, Nov 1992.

[10] D. Middleton, "Statistical-physical models of electromagnetic interference," U.S. Department of Commerce, Office of Telecommunications, Tech. Rep., April 1976.

[11] T. M. Cover and J. A. Thomas, Elements of Information Theory, 2nd ed. Wiley \& Sons, New York, 2006. 